\title{
The Two Phase of the Bayes Statistical Model of Accelerated Test Censoring
}

\author{
Wang Siquan \\ Department of Mathematics of Hong Kong Baptist University, Hong Kong ,999077,China
}

Key Words: Bayesian method; life prediction; distribution model

\begin{abstract}
In the Bayes method, the unknown parameters are given proper prior distribution, and the data are obtained. According to the Bayes theorem, the posterior distribution of the parameters is updated according to the data. In this paper we use Bayesian analysis and get the help of a priori information so that we can avoid the problem which the life can't be estimated caused by a small amount of failure data with the traditional methods. Limited hybrid conjugate prior distribution can represent phase stress prior distribution. We use this concept to make $\mathrm{n}$ test product units be put under the second phase stress (low and high) in turns to test. Then the test won't be stopped until we get a previously scheduled limited time $\mathrm{T}$ and proportion $\pi_{c}$. Our aim is to minimize the asymptotic variance of product life's maximum likelihood estimate in hope of being used normally so as to expect to get the best method. What's more, we can find the best change time $\tau^{*}$ and proportion $\pi^{*}$ among stresses. That is to say, we can get the best life test method limited in Bayesian two phase type I .
\end{abstract}

\section{Introduction}

Here we describe the symbols of gradual limits test model as follows.

$\mathrm{X}_{0}$ : the products' stress level under the normal use

$\mathrm{X}_{\mathrm{i}}$ : the ith gradual stress, $\mathrm{i}=1,2, \ldots, \mathrm{k}, \mathrm{x}_{1}<\mathrm{x}_{2}<\ldots<\mathrm{x}_{\mathrm{k}}$

$\mathrm{n}$ : the total number of test products

$\mathrm{n}_{\mathrm{i}}$ : the number of failure products in the stress $\mathrm{x}_{\mathrm{i}}, \mathrm{i}=1,2, \ldots, \mathrm{k}$

$\mathrm{C}_{\mathrm{i}}$ : the number of gradual limits in the stress $\mathrm{x}_{\mathrm{i}}, \mathrm{i}=1,2, \ldots, \mathrm{k}$

$\mathrm{T}$ : the limited time in gradual limits model

$Y_{\mathrm{ij}}$ : the products' failure time in the stress $x_{i}, i=1,2, \ldots, k, j=1,2, \ldots, n i$

$Y_{i(j)}$ : the order statistics of the products' failure time in the stress $x_{i}, i=1,2, \ldots, k, j=1,2 \ldots n i$

$\lambda_{\mathrm{i}}=\frac{1}{\theta_{\mathrm{i}}}$ :the products' average life in the stress $\mathrm{x}_{\mathrm{i}}, \mathrm{i}=0,1,2, \ldots, \mathrm{k}$

$\pi_{\mathrm{i}}(\cdot)$ :the prior distribution, $\mathrm{i}=1,2, \ldots, \mathrm{k}$ 
$\pi_{\mathrm{i}}\left(\mathrm{y}_{\mathrm{i}, \mathrm{j}}\right)$, the posteriori distribution, $\mathrm{i}=1,2, \ldots, \mathrm{k}, \mathrm{j}=1,2, \ldots, \mathrm{ni}$

$\tau_{\mathrm{i}}:$ the time of gradual stresses change, $\mathrm{i}=1,2, \ldots, \mathrm{k}$

$\pi_{\mathrm{i}}: \mathrm{ni} / \mathrm{n}$, the actual proportion of the test products when the stress is $\mathrm{x}_{\mathrm{i}}, \mathrm{i}=1,2, \ldots, \mathrm{k}$

$\pi_{c_{i}}: \mathrm{nc} / \mathrm{n}$, the proportion of the limited products

$\operatorname{Fi}(\cdot)$ : the cumulative distribution function whose product life is the index distribution and parameter is $\lambda_{i}, \mathrm{i}=1,2, \ldots, \mathrm{k}$

$\mathrm{G}(\cdot)$ : the products' total cumulative distribution function under the gradual limits model of $\mathrm{k}$ phase

$\tau_{V}^{*}, \pi_{V}^{*}:$ the best stress change point according to $\mathrm{V}$-criterion

$\tau_{D}^{*}, \pi_{D}^{*}:$ the best stress change point according to D-criterion

$\mathrm{N}_{\mathrm{i}}: n-\sum_{j=1}^{i-1} n_{j}-\sum_{j=1}^{i-1} c_{j}$, the number of products without failure when the stress xi begins to act, $\mathrm{i}=1,2, \ldots, \mathrm{k}$

Asy $\operatorname{Var}\left(\log \hat{\theta}_{0}\right):$ the gradual variance of the life's maximum likelihood estimate when the product are used normally

\section{Basic Assumptions of Model}

Here we describe the basic assumptions of the gradual limits test model of $\mathrm{k}$ phase as follows.

(1) This model has the stress level of stress gradual limits in $\mathrm{k}$ phase, $x_{i}, \mathrm{i}=1,2, \ldots, \mathrm{k}$. The stresses increase gradually. That is to say, $x_{0}<x_{1}<\cdots x_{k}, x_{0}$ is the stress under the normal use.

(2) For any stress level, the product's life is the index distribution, and the parameter is $\lambda_{i}=\frac{1}{\theta_{i}}, \mathrm{i}=1,2, \ldots, \mathrm{k}$

(3) Under the stress of each phase, there is the logarithm linear function relation between the product's average life and the stress level xi. That is to say, $\log \lambda_{i}=a+b x_{i}, i=1,2, \cdots, k$, among them a and $\mathrm{b}$

$(<0)$ are the unknown parameters. Therefore, the average life of test products under low stress will be longer than the average life under high stress. In the accelerated life test there is the relation of inverse power law model or arrhenius power law model between characteristics and stress for the products which are often assumed. However, the logarithm linear function relation can contain this double model.

(4) This model assumes that the occurrence of effectiveness should depend on cumulative exposure model,cem. That is to say, the remaining life of the gradual limits products only has the relation with the distribution cumulative exposure when it gets the stress at that time. In nelson (1990) it has been discussed in details. 
(5) We can find the appropriate prior information as the prior distribution in the stress under the normal use and the accelerated stress.

\section{Statistical Model}

About the reliability analysis, there are a lot of distributions used to describe the product life, such as Exponential distribution, Gamma distribution, Webb distribution, etc. We assume the relation between failure rate and stress is that there is the logarithm linear function relation between the products' average life and the stress level. That is to say, $\log \lambda_{i}=a+b x_{i}, i=1,2$, among them $\mathrm{x}$ represents the stress, but $\mathrm{a}$ and $\mathrm{b}(<0)$ are unknown parameters. In the formula we can see that parameter $\mathrm{a}$ and $\mathrm{b}$ represent the relation between the failure rate and the stress. The failure rate will have a substantial increase with the increase of experimental stress, and then the value of parameter b will be larger. Conversely, if there is no significant relation between the failure rate and the stress, then the parameter b will be relatively small. However, seen from a certain aspect, a represents the intercept, that is to say, it represents the failure rate without the stress benefit. About the statistical inference of parameter $\mathrm{a}$ and $\mathrm{b}$ traditionally we can use maximum likelihood estimation to estimate. When the sample size is relatively large or the data is relatively complete, usually the maximum likelihood estimation can provide a more accurate result. However, for the test with the small sample or the very insufficient data information, the information with the prior distribution is helpful to the statistical inference of parameters. That is to say, Bayesian method can provide a restively reasonable inference. From hypothesis five and hypothesis two, we select a Gamma conjugate prior distribution for the low stress and the high stress level respectively, for example, the formula (1)

$$
\pi\left(\lambda_{1}, \lambda_{2}\right)=\frac{1}{\Gamma\left(\alpha_{\mathrm{i}}\right) \beta_{\mathrm{i}}^{\alpha_{\mathrm{ii}}}} \lambda_{\mathrm{i}}^{\alpha_{\mathrm{i}}-1} \mathrm{e}^{-\frac{\lambda_{\mathrm{i}}}{\beta_{\mathrm{i}}}}, \mathrm{i}=1,2
$$

And we consider the mixed prior distribution, for example, the formula (2)

$$
\begin{aligned}
\pi\left(\lambda_{1}, \lambda_{2}\right) & =r \cdot \pi_{1}\left(\lambda_{1}, \lambda_{2}\right)+(1-r) \pi_{2}\left(\lambda_{1}, \lambda_{2}\right) \\
& =r \frac{\lambda_{1}^{\alpha_{1}-1} e^{-\frac{\lambda_{1}}{\beta_{1}}}}{\Gamma\left(\alpha_{1}\right) \beta_{1}^{\alpha_{1}}}+(1-r) \frac{\lambda_{2}^{\alpha_{2}-1} e^{-\frac{\lambda_{2}}{\beta_{2}}}}{\Gamma\left(\alpha_{2}\right) \beta_{2}^{\alpha_{2}}}
\end{aligned}
$$

Among them, $\mathrm{r}, 0 \leq \mathrm{r} \leq 1$ is prior decision. That is to say, each mixed part specifies a joint prior distribution to $\lambda_{i}, i=1,2$, in other words, the mixed prior distribution described above means $\lambda_{2}$ is the set prior distribution in the first mixed part. That is to say, $\lambda_{2}$ is the equal distribution in $(0,1)$. However, in the second mixed part $\lambda_{1}$ is the equal distribution in $(0,1)$.

We assume that the tested product $Y_{i j}, \quad i=1,2, j=1,2, \ldots n_{i}$ has the exponential distribution and the parameter is $\lambda_{i}, \mathrm{i}=1,2$,that is to say, it is product failure rate. Then, the probability density function of $Y_{i j}$, is $f_{i}\left(y_{i j}\right)=\lambda_{i} e^{-\lambda_{i, y_{i}}}, \mathrm{i}=1,2, \mathrm{j}=1,2, \ldots$ ni. According to the hypothesis four, the occurrence of this 
model failure is based on the cumulative exposure mode, then the cumulative distribution function of the failure of the Bayesian two -phase products can be written as (3)

$$
G(y)=\left\{\begin{array}{cc}
F_{1}(y), & 0 \leq y \leq \tau \\
F_{2}(y+s-\tau) & \tau \leq y \leq T
\end{array} \quad F_{2}(s)=F_{1}(\tau)\right.
$$

and the likelihood function is

$$
L\left(\lambda_{1}, \lambda_{2}\right)=\prod_{j=1}^{n_{1}}\left[\lambda_{1} e^{-\lambda_{y_{1}}}\right] \cdot \prod_{j=1}^{n_{2}}\left[\lambda_{2} e^{-\lambda_{2}\left(y_{2}(j)-\tau\right)-\lambda_{1} \tau}\right] \prod_{j=1}^{n_{c}}\left[e^{-\lambda \tau-\lambda_{2}\left(T-\tau_{1}\right.}\right]
$$

Among them, $n_{c}=n-n-n_{2}, \tau$ is the stress change point and $\mathrm{T}$ is the limited time.

Therefore, the product of the mixed posterior distribution of $Y_{i j}, i=1,2, j=1,2, \ldots$ ni is the formula

(2) and formula (4) is described as the formula (5)

$$
\begin{aligned}
& \pi\left(\lambda_{1}, \lambda_{2} \mid y_{i, j}, i=1,2 ; j=1,2, \cdots n_{i}\right)
\end{aligned}
$$

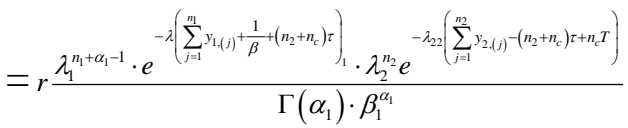

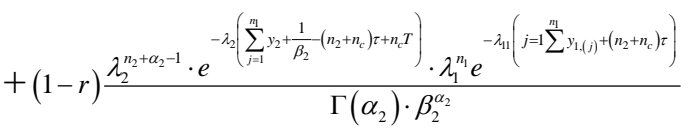

We make $\pi\left(\lambda_{1}, \lambda_{2} \mid y_{i, j}, i=1,2 ; j=1,2, \cdots n_{i}\right)$ integrate $\lambda_{\mathrm{i}}, \mathrm{i}=1,2$ Individually, so the mixed marginal posterior distributions of $\lambda_{\mathrm{i}}, \mathrm{i}=1,2$ are the formula (6) and the formula (7) respectively

$$
\begin{aligned}
& \pi\left(\lambda_{1} \mid y_{i, j}, i=1,2 ; j=1,2, \cdots n_{i}\right) \\
& =r \cdot \Gamma\left(n_{1}+\alpha_{1},\left(U_{1}+\frac{1}{\beta_{1}}\right)^{-1}\right)+(1-r) \cdot \Gamma\left(n_{1}+1,\left(U_{1}\right)^{-1}\right) \\
& \pi\left(\lambda_{2} \mid y_{i, j}, i=1,2 ; j=1,2, \cdots, n_{i}\right) \\
& =r \cdot \Gamma\left(n_{2}+1,\left(U_{2}\right)^{-1}\right)+(1-r) \cdot \Gamma\left(n_{2}+\alpha_{2},\left(U_{2}+\frac{1}{\beta_{2}}\right)^{-1}\right)
\end{aligned}
$$

Among them

$$
U_{1}=\sum_{j=1}^{n_{1}} y_{1,(j)}+\left(n_{2}+n_{2}\right) \tau, \quad\left(n-n_{2}\right) \tau \leq U_{1} \leq n \tau
$$

and

$$
\begin{aligned}
& U_{2}=\sum\left(y_{2,(j)}-\tau\right)+(T-\tau) n_{c} \\
& n_{c} T-\left(n-n_{1}\right) \tau \leq U_{2} \leq\left(n-n_{1}\right) T,
\end{aligned}
$$

are the total test time of the products when the stress is $x i, i=1,2$. 
In Bayesian inference, if we want to measure the advantages and disadvantages of the estimation, usually we require to consider the accuracy and precision of the estimation. Generally speaking, the most commonly used method is the sum of squares. Therefore, we use the formula (6) and the formula (7), namely the expected value of the mixed marginal posterior distribution when $\lambda_{i}$ is in the stress $\mathrm{x}_{\mathrm{i}}, \mathrm{i}=1,2$, to estimate $\lambda_{i}, \mathrm{i}=1,2$, and they can be described as the formula ( 8 ) and the formula

(9) respectively.

$$
\begin{aligned}
& \hat{\lambda}_{1}=r \frac{n_{1}+\alpha_{1}}{U_{1}+\frac{1}{\beta_{1}}}+(1-r) \cdot \frac{n_{1}}{U_{1}} \\
& \hat{\lambda}_{2}=r \cdot \frac{n_{2}+1}{U_{2}}+(1-r) \frac{n_{2}+\alpha_{2}}{U_{2}+\frac{1}{\beta_{2}}}
\end{aligned}
$$

Practically we also often consider the Bayesian estimation of $\lambda_{i}, \mathrm{i}=1,2$ includes the mode and median of the formula (6) and the formula (7). If possible, we should compare the differences among the three kinds of things mentioned above at the same time, especially when the prior distribution has changed, we should compare the robustness among them.

According to the basic hypothesis three we put $\log \lambda_{i}=a+b_{i}$ into $\lambda_{i}, \mathrm{i}=1,2$ in the formula. Then the $\log$ likelihood function of $Y_{i j},=y i, j, i=1,2, j=1,2, \ldots$ ni is the function of unknown parameter a and the parameter b, for example, the formula (10)

$$
\begin{aligned}
& \ln \pi\left(\lambda_{1}, \lambda_{2} / y_{i, j}, i=1,2, j=1,2, \cdots, n_{i}\right) \\
& =\left(2 n_{1}+2 n_{2}+\alpha_{1}+\alpha_{2}-2\right) a+\left[\left(2 n_{1}+\alpha_{1}-1\right) x_{1}+\left(2 n_{2}+\alpha_{2}-1\right) x_{2}\right] b \\
& -\left(2 U_{1}+\frac{1}{\beta_{1}}\right) e^{a+b x}-\left(2 U_{2}+\frac{1}{\beta_{2}}\right) e^{a+b x} \\
& +\ln \left(\frac{r(1-r)}{\Gamma\left(\alpha_{1}\right) \Gamma\left(\alpha_{2}\right) \beta_{1}^{\alpha_{2}} \beta_{2}^{\alpha_{1}}}\right)
\end{aligned}
$$

Then the maximum likelihood estimation of $\hat{a}$ and $\hat{b}$ can be obtained from the following two formulas.

$$
\begin{aligned}
& \frac{\partial \ln L\left(y_{i, j} ; a, b\right)}{\partial a}=-\sum_{i=1}^{k}\left(n_{i}+\alpha_{i}-1\right)+\sum_{i=1}^{k}\left(2 U_{i}+\frac{1}{\beta_{i}}\right) e^{\left(a+b x_{i}\right)}=0 \\
& \frac{\partial \ln L\left(y_{i, j} ; a, b\right)}{\partial b}=-\sum_{i=1}^{k}\left(2 n_{i}+\alpha_{i}-1\right) x_{i}+\sum_{i=1}^{k}\left(2 U_{i}+\frac{1}{\beta_{i}}\right) x_{i} e^{\left(a+b x_{i}\right)}=0
\end{aligned}
$$

Namely,

$$
\hat{a}=\log \left(\frac{\sum_{i=1}^{k}\left(2 U_{i}+\frac{1}{\beta_{i}}\right) e^{\hat{b x}}}{\sum_{i=1}^{k}\left(2 n_{i}+\alpha_{i}-1\right)}\right)
$$


and $\left[\sum_{i=1}^{k}\left(2 n_{i}+\alpha_{i}-1\right)\right]\left[\sum_{i=1}^{k}\left(2 U_{i}+\frac{1}{\beta_{i}}\right) x_{i} e^{\hat{b}^{x_{i}}}\right]-\left[\sum_{i=1}^{k}\left(2 n_{i}+\alpha_{i}-1\right) x_{i}\right]\left[\sum_{i=1}^{k}\left(2 U_{i}+\frac{1}{\beta_{i}}\right) \hat{e}^{\hat{b}_{i}}\right]=0 \quad$ We use $l=\ln \pi\left(\lambda_{1}, \lambda_{2} \mid y_{i, j}, i=1,2 ; j=1,2, \cdots n_{i}\right)$ to make the partial derivation of the parameter a and the parameter b, so we can get

$$
\begin{aligned}
& E\left(\frac{\partial^{2} l}{\partial a^{2}}\right)=-E\left[\left(2 U_{1}+\frac{1}{\beta_{1}}\right) \lambda_{1}+\left(2 U_{2}+\frac{1}{\beta_{2}}\right) \lambda_{2}\right] \\
& E\left(\frac{\partial^{2} l}{\partial b^{2}}\right)=-E\left[\left(2 U_{1}+\frac{1}{\beta_{1}}\right) x_{1}^{2} \lambda_{1}+\left(2 U_{2}+\frac{1}{\beta_{2}}\right) x_{2}^{2} \lambda_{2}\right] \\
& E\left(\frac{\partial^{2} l}{\partial a b}\right)=-E\left[\left(2 U_{1}+\frac{1}{\beta_{1}}\right) x_{1} \lambda_{1}+\left(2 U_{2}+\frac{1}{\beta_{2}}\right) x_{2} \lambda_{2}\right] \\
& E\left(U_{1} \lambda_{1}\right)=n\left\{\left[1-r\left(1+\beta_{1} \tau\right)^{-\alpha_{1}}+(1-r) \tau^{-1}\left(1-e^{-\tau}\right)\right]\right\} \\
& E\left(U_{2} \lambda_{2}\right)=n\left\{r\left(1+\beta_{1} \tau\right)^{-\alpha}\left[1-(T-\tau)^{-1}\left(1-e^{-(T-\tau)}\right)\right]+(1-r) \tau^{-1}\right. \\
& \left.\left(1-e^{-\tau}\right) \times\left[1-\left(1+\beta_{2}(T-\tau)\right)^{-\alpha}\right]\right\}
\end{aligned}
$$

Among them

$$
\begin{aligned}
& E\left(\frac{N_{1}}{n} / \lambda_{1}\right)=\int_{0}^{\tau} \lambda_{1} e^{-\lambda_{1} y} d y=1-e^{-\lambda_{1} \tau} \\
& E\left(\frac{N_{2}}{n} / \lambda_{2}\right)=\int_{\tau}^{T} \lambda_{2} e^{-\lambda_{2}\left(y-\frac{\lambda_{2}-\lambda_{1}}{\lambda_{2}} \tau\right)} d y=e^{-\lambda_{1} \tau}\left(1-e^{-\lambda_{2}(T-\tau)}\right) \\
& E\left[\sum_{j=1}^{N_{1}} Y_{1,(j)} / N_{1}, \lambda_{1}\right]=N_{1} E\left[Y_{1, j}^{*} / N_{1}, \lambda_{1}\right]=N_{1}\left(\frac{1}{\lambda_{1}}-\frac{\tau e^{-\lambda_{1} \tau}}{1-e^{-\lambda_{1} \tau}}\right) \\
& E\left[\sum_{j=1}^{N_{2}} Y_{2,(j)} / N_{2}, \lambda_{2}\right]=N_{2} E\left[Y_{2, j}^{*} / N_{2}, \lambda_{2}\right]=N_{2}\left(\frac{\left(\tau+\lambda_{2}^{-1}\right)-e^{-\lambda_{2}(T-\tau)}\left(T+\lambda_{2}^{-1}\right)}{1-e^{-\lambda_{2}(T-\tau)}}\right)
\end{aligned}
$$

In the main formula (18) and (19), when the stress is $\mathrm{x} 1$ respectively, $Y_{1, j}^{*}$ is the truncated distribution and it is in the interval $(0, \tau)$. However, when the stress is $\mathrm{x} 2, Y_{2, j}^{*}$ is the truncated distribution and it is in the interval $(\tau, \mathrm{T})$. And the distribution of $N_{1} / \lambda_{1}$ is $B\left(n, 1-e^{-\lambda_{1} \tau}\right)$ and the distribution of $N_{2} / \lambda_{2}$ is $B\left(n, e^{-\lambda_{1} \tau}\left(1-e^{-\lambda_{2}(T-\tau)}\right)\right)$.

Set

$$
E\left(U_{1} \lambda_{1}\right) / n=A_{1}(\tau)
$$

and 


$$
E\left(U_{2} \lambda_{2}\right) / n=A_{2}(\tau)
$$

Here $A_{i}(\tau)$ are the probability of failure when the stress of product is $x i, i=1,2$ respectively. Therefore, the Fisher information matrix I is

$$
I=\left[\begin{array}{l}
\sum_{i=1}^{2}\left[2 n A_{i}(\tau)+a_{i}(r)\right], \sum_{i=1}^{2}\left[2 n A_{i}(\tau)+a_{i}(r)\right] x_{i} \\
\sum_{i=1}^{2}\left[2 n A_{i}(\tau)+a_{i}(r)\right] x_{i}, \sum_{i=1}^{2}\left[2 n A_{i}(\tau)+a_{i}(r)\right] x_{i}^{2}
\end{array}\right]
$$

Among them

$$
a_{1}(r)=E\left(\frac{\lambda_{1}}{\beta_{1}}\right)=\beta_{1}^{-1}\left[r \alpha_{1} \beta_{1}+(1-r) \cdot \frac{1}{2}\right]
$$

and

$$
a_{2}(r)=E\left(\frac{\lambda_{2}}{\beta_{2}}\right)=\beta_{2}^{-1}\left[r \cdot \frac{1}{2}+(1-r) \alpha_{2} \beta_{2}\right]
$$

So the inverse matrix $I^{-1}$ of matrix $I$ is

$$
I^{-1}=\left[\begin{array}{l}
\sum_{i=1}^{2}\left[2 n A_{i}(\tau)+a_{i}(r)\right] x_{i}^{2},-\sum_{i=1}^{2}\left[2 n A_{i}(\tau)+a_{i}(r)\right] x_{i} \\
-\sum_{i=1}^{2}\left[2 n A_{i}(\tau)+a_{i}(r)\right] x_{i}, \sum_{i=1}^{2}\left[2 n A_{i}(\tau)+a_{i}(r)\right]
\end{array}\right]
$$

Therefore, the variance of maximum likelihood estimation $A s y \cdot \operatorname{Var}_{x}\left(\log \hat{\lambda}_{0}\right)$ of life value when the products are used normally is

$$
\begin{gathered}
\operatorname{Asy} \cdot \operatorname{Var}_{x}\left(\log \hat{\lambda}_{0}\right)=\operatorname{Asy} \cdot \operatorname{Var}_{x}(\hat{a})+2 x_{0} \operatorname{Ass} \cdot \operatorname{Cov}_{x}(\hat{a}, \hat{b}) \times \\
x_{0}^{2} \operatorname{Asy} \cdot \operatorname{Var}_{x}(\hat{b})
\end{gathered}
$$

Among them $\operatorname{Var}_{x}(\hat{a}), \operatorname{Cov}_{x}(\hat{a}, \hat{b})$ and $\operatorname{Var}_{x}(\hat{b})$ can be obtained from the formula.

From the formulas $(20)-(22)$, we can apparently get that $A s y \cdot \operatorname{Var}_{x}\left(\log \hat{\lambda}_{0}\right)$ only connect with the stress $x_{i}, \mathrm{i}=1,2$, and the time change point $\tau$ among the stresses. In order to minimize Asy. $\operatorname{Var}_{x}\left(\log \hat{\lambda}_{0}\right)$, we make $A s y \cdot \operatorname{Var}_{x}\left(\log \hat{\lambda}_{0}\right)$ get the partial differential on $\tau$ to get the best time change point $\tau^{*}$ of the best method, that is to say, we set

$$
\frac{\partial A s y \cdot \operatorname{Var}_{x}\left(\log \hat{\lambda}_{0}\right)}{\partial \tau}=0 \text { 。 }
$$




\section{Conclusion}

We mainly introduce the pressurized best life test method of limited phase about Bayesian type I and analyze the theory at present in the paper. At the same time we also introduce some basic concepts and terminologies in the field. In this way, it riches the statistical inference of gradual accelerated exponential life in the theory and provides the basis for the related practical research.

\section{References}

[1] balakrishnan,N.and Han,D.optimal step-stress testing for progressively Type-I censored data from exponential distribution [J], . statist. Plann.Inference to appear. 2008

[6] Fan T.H W.L.and Balakrishnan,N .Exponential progressive step-stress life-testing with link function based on Box-Cox transformation[J]. Journal of statistical Planning and inference 2008,138,2340-2354.

[10] Xiong,C.and Ji,M. Analysis of grouped and censored data from step-stress life test [J].IEEE Transactions on Reliability , 2004,53,22-28

[11] Wu,S.J. ,Lin,Y. P.and Chen,Y.J. Planning step-stress life test with progressively type- I group-censored exponential data [J]. Neerlandica,2006,60,46-56

[15] Gu Quanlong, Zhou Xiaodong,etc. The Bayes Statistical Analysis of Constant-stress Accelerated Life Test Missing Data under Exponential Distribution Situation[J]. Applied Mathematics:A Journal of Chinese Universities(Series A), 2006,21(2):183-190.

[43] Wu, S. J., Chang, C. T.. Inference in the Pareto distribution based on progressive Type II censoring with random removals[J]. J. Appl. Statist. 2003, 30(2):163-172. 